\title{
Économie du sport professionnel et équilibre compétitif. Les limites d'une approche largement utilisée : « Noll-Scully measure of Competitive Balance »
}

\author{
Nicolas Scelles ${ }^{1}$, Christophe Durand ${ }^{2}$ \\ ${ }^{1}$ Centre de Recherche en Gestion (EA 1722), Université de Poitiers, Faculté des Sciences du Sport, 8 allée Jean Monnet, \\ 86000 Poitiers, France \\ 2 Centre d'Étude Sport et Actions Motrices (EA 4260), Université de Caen, UFR STAPS, Campus II, Bd du Maréchal Juin, \\ Caen Cedex, 14032, France
}

Reçu le 8 juillet 2011 - Accepté le 9 décembre 2011

\begin{abstract}
Résumé. L'équilibre compétitif constitue un concept fondateur de l'économie du sport professionnel. Il postule un lien central entre l'incertitude du résultat et la demande du public. La mesure la plus utilisée dans la littérature est l'approche « Noll-Scully ». Elle confronte l'écart dans le classement des équipes entre le réel observé et le théorique idéal compte tenu du nombre de matchs. L'objet de cette contribution est de discuter la pertinence de l'approche. Après un aperçu de la littérature, la $2^{\mathrm{e}}$ partie aborde les points d'achoppement que sont son objectif (intégrer l'influence du nombre de matchs) et sa formulation (incorporer une valeur théorique idéale). L'article oriente ensuite la discussion sur une mesure alternative susceptible d'éviter les lacunes de l'approche « Noll-Scully ».
\end{abstract}

Mots clés : Économie du sport professionnel, équilibre compétitif, incertitude du résultat, demande, approche «Noll-Scully »

\begin{abstract}
Professional sports economics and competitive balance. Limits of a very used approach: "Noll-Scully measure of Competitive Balance".

Competitive balance constitutes a founder concept of professional sports economics. It postulates a central link between uncertainty of outcome and public demand. The most used measure in the literature is "Noll-Scully" approach. It confronts teams spread in the standing between observed reality and theoretical ideal in taking into account the number of matches. The object of this contribution is to discuss "Noll-Scully" approach pertinence. Following a summary of the literature, the 2nd part approaches the obstacle points that are its purpose (to integrate the influence of the number of matches) and its formulation (to incorporate a theoretical ideal value). Then the article positions the discussion on an alternative measure able to avoid the gaps of "Noll-Scully" approach.
\end{abstract}

Key words: Professional sports economics, competitive balance, uncertainty of outcome, demand, "NollScully" approach

\section{Introduction}

L'équilibre compétitif (EC, competitive balance) constitue un concept fondateur de l'économie du sport professionnel (Andreff, 2008, 2009 ; Neale 1964 ; Rottenberg, 1956). Il se définit dans la littérature comme la recherche d'une relative égalité des forces sportives, génératrice d'une incertitude du résultat, celle-ci étant postulée favorable à la demande du public et des médias. La recherche d'EC est à la base de la doctrine nord-américaine d'exception sportive qui a généré un mode de régulation spécifique à ce secteur, via par exemple le Sports Broadcasting Act voté par le Congrès en 1961. L'exemption aux lois anti-trust de la négociation des droits TV nationaux par les ligues (et non les clubs) est assimilée à un cartel légal. Elle se justifie par les bénéfices supposés du partage à parts égales de ces droits entre clubs (Gouguet, 2004). Un tel partage est censé favoriser l'EC, ce qu'une approche pure et dure par la concurrence entre les clubs ne permettrait pas. En effet, le marché creuse les écarts et déséquilibre le championnat financièrement et sportivement, ce qui nuit aux équipes dominées 
- effet attendu - mais aussi aux équipes dominantes effet spécifique au sport (Neale, 1964).

De nombreux travaux se sont penchés sur l'EC, proposant différentes mesures de l'incertitude du résultat. Dans la littérature académique, l'EC est étudié à travers quatre échelles temporelles d'incertitude :

1) sur l'issue des matchs ;

2) en cours de saison ;

3) au terme d'une saison ;

4) sur plusieurs exercices afin d'établir s'il y a domination régulière d'une ou quelques équipes (Szymanski, 2003, pp. 1153-1156).

La structure de l'article comprend trois parties. Dans une première partie, il s'agit de réaliser une revue de littérature des travaux sur la mesure de l'EC afin de rendre compte de la diversité des approches possibles. La partie suivante s'attache à expliquer pourquoi la mesure la plus utilisée dans la littérature - l'approche 《Noll-Scully » (Noll-Scully measure of Competitive Balance, NSCB ; Noll, 1988 ; Scully, 1989) - pose problème du point de vue de sa construction. Une discussion sur la mesure à préconiser est ensuite envisagée, permettant de faire état de la difficulté à déterminer la «bonne » mesure.

\section{Les mesures de l'équilibre compétitif dans la littérature et ses quatre niveaux temporels}

\subsection{L'incertitude sur l'issue des matchs}

Contrairement aux trois niveaux suivants qui correspondront à des mesures ex-post donc sur l'incertitude réelle, l'incertitude sur l'issue des matchs constitue une mesure ex-ante donc sur l'incertitude anticipée. Dans The economic design of sports contests (2003), Szymanski (pp. 1157-1158) résume sous forme de tableau les recherches sur l'incertitude du résultat en tant que facteur explicatif de la demande. En 1975, Hart, Hutton et Sharot proposent la différence logarithmique des positions au classement pour traduire l'incertitude sur l'issue des matchs. Le tableau réalisé par Szymanski ne fait pas mention de l'étude économétrique de Siegfried et Hinshaw de 1979. Pourtant, cette analyse intègre le niveau de concurrence entre équipes, appréhendé à travers la différence de rangs au classement, mesure reprise en 2002 par Garcìa et Rodrìguez et en 2006 par Pinnuck et Potter. En 1988, Jones et Ferguson ont retenu l'appartenance des équipes au haut ou au bas du classement. Or, cette mesure, au même titre que les précédentes, ne prend pas en compte certains aspects comme par exemple l'état de forme des équipes qui s'affrontent ou quelle équipe reçoit : l'avantage du jeu à domicile, home advantage, a fait l'objet de nombreux travaux en psychologie du sport (Dosseville, 2007) et a été intégré récemment par des économistes du sport (Forrest, Simmons \& Buraimo, 2005 ;
Forrest \& Simmons, 2006 ; Baranzini, Ramirez \& Weber, 2008).

Dans son état de la littérature, Szymanski relève que, depuis 1988, les études relatives à l'incertitude sur le dénouement des matchs se sont largement appuyées sur les cotes des équipes (fixing) dans le cadre des jeux de paris (Forrest \& Simmons, 2002 ; Knowles, Sherony \& Haupert, 1992 ; Kuypers, 1996 ; Peel \& Thomas, 1988, 1992, 1997 ; Rascher, 1999). Pour compléter le propos, on peut ajouter les analyses de Czarnitzki et Stadtmann (2002) ainsi que d'Owen et Weatherston (2004), non relevées par Szymanski (Tab. 1). Les cotes des équipes présentent l'avantage d'intégrer les points manquants dans les mesures antérieures, ce qui justifie peut-être le fait qu'elles soient fortement utilisées à présent. On notera qu'au sein de leur modèle, Peel et Thomas (1997) emploient de telles cotes mais avec une particularité : elles prennent en compte l'écart de points au score en attribuant un handicap à l'équipe favorite.

\subsection{L'incertitude en cours de saison}

Le tableau de Szymanski (2003) mentionne un certain nombre d'études intégrant des mesures d'incertitude en cours de saison. Toutefois, l'analyse fondatrice de Hill, Madura et Zuber de 1982 n'y figure pas. Les auteurs s'appuient pour chaque match sur le nombre de matchs à gagner pour rejoindre le leader pour les deux équipes concernées.

En 1984, Jennett prend en compte le degré d'incertitude pour chaque équipe match après match en fonction de la possibilité arithmétique pour ces équipes d'être rejointes ou de rattraper celle classée première ou celles relégables. Cette possibilité arithmétique dépend du nombre de matchs restant à jouer. Andreff $(2008$, p. 18) illustre cet état de fait : «Par exemple, en Ligue 1, à cinq journées de la fin du championnat, toute équipe à 15 points ou moins ( 5 victoires $=15$ points) du leader est encore en course pour le titre, à trois journées de la fin seules restent les équipes à 9 points ou moins du leader, et ainsi de suite. » Néanmoins, la perspective qu'un club classé à 15 points du premier à cinq journées de la fin puisse le rejoindre est difficilement envisageable! Comme le note Andreff : «Cette étude présente l'inconvénient d'être basée sur les possibilités arithmétiques d'être encore champion là où il faudrait à l'évidence une approche probabiliste. »

En 1987, Cairns propose une méthode a priori plus réaliste. En effet, l'auteur pose le principe suivant : «Une équipe est encore en course (in contention) si elle peut remporter le titre en gagnant $80 \%$ des points lors des matchs qui lui restent à jouer, en faisant l'hypothèse que le leader ne gagnera que $50 \%$ de ses derniers matchs » (Andreff, 2008, p. 18). À l'inverse de précédemment, cette approche amène à considérer comme sans incertitude des situations en réalité incertaines. Ainsi, un retard de trois points à deux journées de la fin paraît résorbable. Or, en 
Tableau 1. Études économétriques intégrant l'incertitude sur l'issue des matchs.

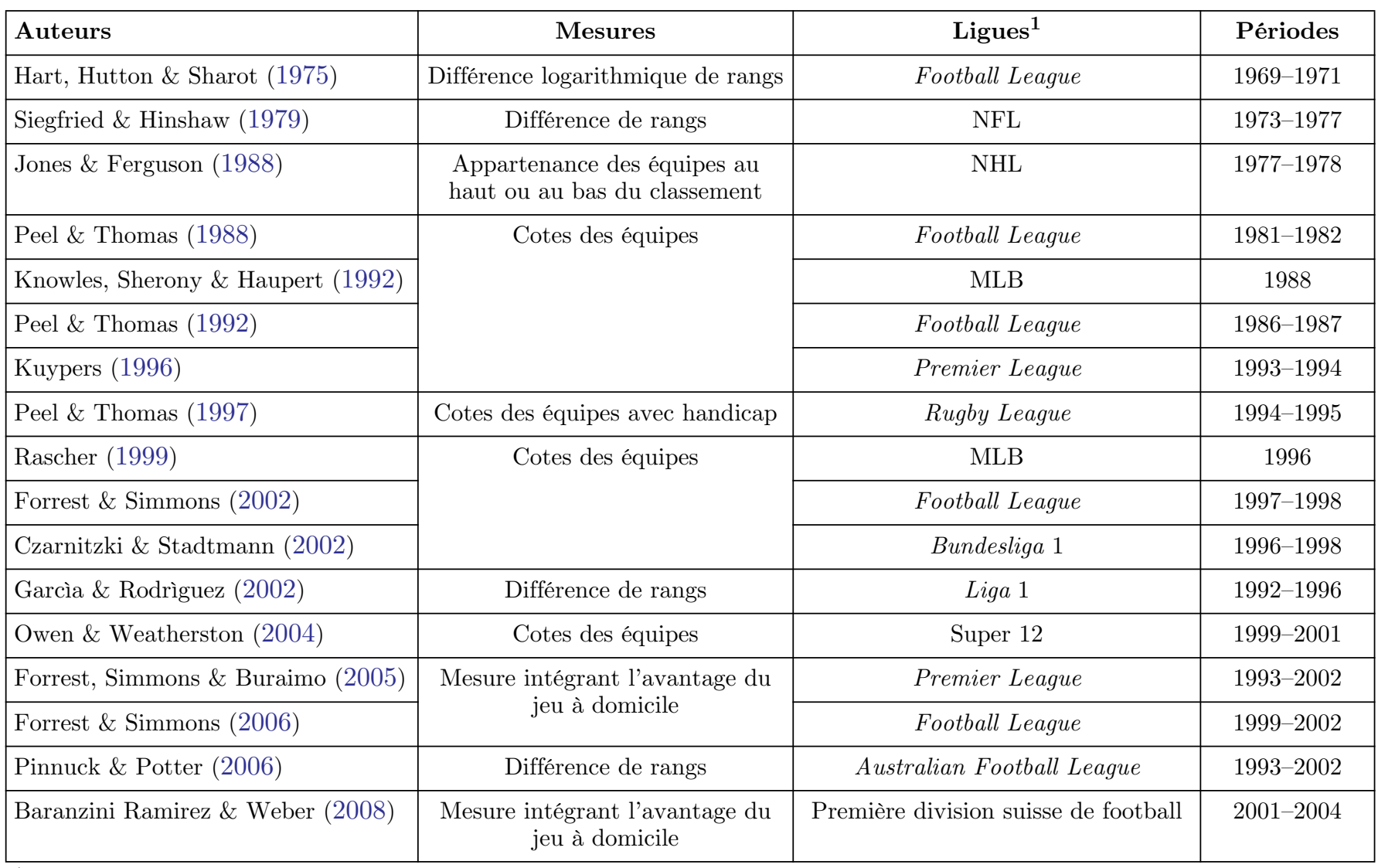

${ }^{1}$ La signification des sigles et plus généralement le niveau, l'espace et le sport des championnats figurant dans les tableaux sont fournis en annexe (sauf quand l'intitulé est suffisamment explicite).

appliquant la méthode de Cairns, on est conduit à exclure cette possibilité puisque l'équipe «suiveuse » prend cinq points contre trois pour le leader, ce qui ne permet pas de combler le retard.

En 1987 également, Borland calcule quatre mesures : 1) la différence de matchs gagnés entre le premier et le dernier, c'est-à-dire l'amplitude ;

2) la somme des coefficients de variation des victoires afin de renseigner sur la dispersion de ces dernières ;

3) le nombre moyen de matchs à gagner pour rejoindre le leader pour l'ensemble des équipes ;

4) le nombre d'équipes à un ou deux matchs au plus des places qualificatives pour les playoffs (mesure pouvant être considérée comme une variante de la précédente même si on s'attache aux places qualificatives pour les playoffs et non plus à la première).

\footnotetext{
$\overline{1}$ Tours à élimination directe concernant les équipes les mieux classées lors de la saison régulière afin de déterminer le champion.
}

Il s'agit de mesures d'incertitude en cours de saison que Borland applique à quatre moments de la saison. Cette approche marque une évolution sensible de la doctrine de la mesure de l'EC.

D'autres mesures d'incertitude en cours de saison seront introduites par la suite (Tab. 2) mais une seule sera reprise par d'autres auteurs : celle de Kuypers (1996) qui croise le nombre de matchs restants et l'écart de points avec le leader à travers le produit de ces deux éléments. Cette mesure sera utilisée par Garcìa et Rodrìguez en 2002, Pinnuck et Potter en 2006 et Baranzini et al. en 2008.

\subsection{L'incertitude du résultat/I'équilibre compétitif au terme d'une saison}

Dans la littérature, une distinction est opérée entre les mesures d'incertitude du résultat et celles d'EC au terme d'une saison (Brandes \& Franck 2007, p. 379 ; Fort \& Maxcy, 2003, p. 155 ; Soebbing, 2008, p. 119) :

1) les premières sont utilisées comme représentant une variable parmi d'autres dans les modèles économétriques 
Tableau 2. Études économétriques intégrant l'incertitude en cours de saison.



d'estimation des affluences et peuvent concerner aussi bien une équipe que la ligue complète ;

2) les secondes sont le plus souvent calculées pour évaluer l'évolution du degré de concurrence sportive au sein de la ligue, particulièrement suite à un changement de règle.

\subsubsection{Les mesures utilisées dans les modèles économétriques d'estimation des affluences}

L'analyse de Demmert de 1973, omise par Szymanski en 2003, utilise un indice évaluant le degré de lutte pour le titre, le nombre de victoires de retard sur le leader pour une équipe et l'écart moyen entre les équipes premières et deuxièmes. Szymanski relève pour sa part la contribution de Noll de 1974. L'auteur s'appuie, comme Demmert, sur le nombre de matchs gagnés de retard sur le leader pour une équipe. En outre, Noll incorpore une variable d'incertitude pour la qualification d'une équipe en playoffs si cette équipe n'a pas plus de cinq points d'avance sur le premier non qualifié pour les playoffs ou de retard sur le dernier qualifié pour les playoffs en fin de saison régulière. D'autres mesures d'incertitude au terme d'une saison seront introduites dans des modèles économétriques par la suite (Tab. 3).

\subsubsection{Les mesures utilisées pour évaluer l'évolution du degré de concurrence}

Dans le cadre de cette seconde utilisation, le recours au tableau de Szymanski n'est plus opportun puisqu'y figurent des mesures «d'incertitude du résultat » et non «d'équilibre compétitif ». En 1981, Daly et Moore proposent l'amplitude et le coefficient de variation des pourcentages de victoires. Quirk et Fort (1992), La Croix et Kawaura (1999) et Andreff (2008, 2009) s'appuient également sur l'amplitude. On peut relever que le coefficient de variation est égal à l'écart-type divisé par la moyenne. Or, lorsque l'on considère les pourcentages de 
Tableau 3. Études économétriques intégrant l'incertitude au terme d'une saison.

\begin{tabular}{|c|c|c|c|}
\hline Auteurs & Mesures & Ligues & Périodes \\
\hline \multirow[t]{3}{*}{ Demmert (1973) } & $\begin{array}{c}\text { Indice évaluant le degré de lutte pour le } \\
\text { titre }\end{array}$ & \multirow[t]{3}{*}{ MLB } & \multirow[t]{3}{*}{$1951-1969$} \\
\hline & $\begin{array}{c}\text { Nombre de matchs gagnés de retard sur le } \\
\text { leader }\end{array}$ & & \\
\hline & $\begin{array}{c}\text { Écart moyen entre les équipes premières et } \\
\text { deuxièmes }\end{array}$ & & \\
\hline \multirow[t]{2}{*}{ Noll (1974) } & $\begin{array}{c}\text { Nombre de matchs gagnés de retard sur le } \\
\text { leader }\end{array}$ & MLB & $1970-1971$ \\
\hline & $\begin{array}{c}\text { Variable d'incertitude pour la qualification } \\
\text { d'une équipe en playoffs }\end{array}$ & NHL & $1972-1973$ \\
\hline Whitney (1988) & \begin{tabular}{|} 
Probabilité moyenne attendue de gagner le \\
championnat compte tenu du classement la \\
saison précédente
\end{tabular} & MLB & $1970-1984$ \\
\hline Schmidt \& Berri (2001) & $\begin{array}{c}\text { Coefficient de Gini des pourcentages de vic- } \\
\text { toires }\end{array}$ & MLB & $\begin{array}{c}1903-1998 \text { et } \\
1975-1988\end{array}$ \\
\hline \multirow[t]{2}{*}{ Humphreys (2002) } & Écart-type des pourcentages de victoires & \multirow[t]{2}{*}{ MLB } & \multirow{2}{*}{$\begin{array}{c}\text { 1901-1999 } \\
\text { (moyenne sur } \\
\text { cinq ans) }\end{array}$} \\
\hline & Indice d'Herfindahl & & \\
\hline Berri, Schmidt \& Brook (2004) & NSCB & NBA & $1992-1996$ \\
\hline Brandes \& Franck (2007) & $\begin{array}{l}\text { NSCB version Cain et HaddockIndice de } \\
\text { concentration de points des premiers } \\
\text { Indice d'Herfindahl }\end{array}$ & $\begin{array}{c}\text { Bundesliga } 1 \text { Premier League } \\
\text { Championship Division, Serie A, } \\
\text { Serie B, Ligue } 1\end{array}$ & $1963-2006$ \\
\hline \multirow[t]{2}{*}{ Soebbing (2008) } & $\begin{array}{c}\text { Nombre de matchs gagnés de retard sur les } \\
\text { places qualificatives pour les playoffs }\end{array}$ & \multirow[t]{2}{*}{ MLB } & \multirow[t]{2}{*}{$1920-2006$} \\
\hline & NSCB & & \\
\hline
\end{tabular}

victoires, leur moyenne à l'échelle d'une ligue sans match nul est égale à 0,5 . Par conséquent, le coefficient de variation des pourcentages de victoires est égal à deux fois l'écart-type des pourcentages de victoires. Ce dernier, ou l'écart-type du pourcentage de points, a souvent été retenu (Tab. 4). En 1988, Noll suggère une approche permettant d'intégrer l'influence du nombre de matchs sur l'écart-type des pourcentages de victoires. Il s'agit du rapprochement entre la mesure effective de l'écart-type des pourcentages de victoires de la saison avec sa valeur théorique représentative d'un championnat parfaitement équilibré, tout en tenant compte du nombre de rencontres. Un an plus tard, Scully applique cette méthode, d'où l'appellation d'approche « Noll-Scully » ou NSCB (Quirk \& Fort, 1992, p. 244). Celle-ci est très usitée dans la littérature à tel point qu'on peut parler de quasi consensus quant au statut de mesure idéale pour la NSCB parmi les économistes du sport. Michie et Oughton ont néanmoins dès 2004 opposé des arguments au bien fondé de la NSCB.

\subsection{L'équilibre compétitif sur plusieurs saisons}

Les mesures d'EC au terme d'une saison sont globalement transposables à celles sur plusieurs saisons. Il est effectivement envisageable de réaliser des calculs à partir des pourcentages de victoires ou de points pris par chaque équipe dans une perspective pluriannuelle. Néanmoins, cela suggère la fermeture de la ligue (nécessité de disposer des mêmes équipes), sauf dans deux cas de figure :

1) si on se cible sur la distribution des pourcentages de victoires ou de points indépendamment des équipes concernées (Quirk \& Fort, 1992);

2) pour les indices de concentration des pourcentages de victoires ou de points des meilleures équipes que l'on peut également appliquer aux titres remportés sur une période (Quirk \& Fort, 1992 ; Scully, 1989 ; Szymanski \& Kuypers, 1999).

D'autres calculs permettent d'appréhender la proximité éventuelle des rangs des équipes d'une saison sur 
Tableau 4. Études intégrant l'équilibre compétitif au terme d'une saison.




Tableau 5. Études intégrant l'équilibre compétitif sur plusieurs saisons.

\begin{tabular}{|c|c|c|c|}
\hline Auteurs & Mesures & Ligues & Périodes \\
\hline Rottenberg (1956) & Répartition des titres & MLB & $1920-1951$ \\
\hline Jones) & Rangs des équipes & NHL & $1946-1966$ \\
\hline Daly \& Moore (1981) & Répartition des titres & MLB & $1955-1973$ \\
\hline Bourg (1989) & Répartition des titres & Ligue 1 & $1946-1988$ \\
\hline Quirk \& Fort (1992) & $\begin{array}{c}\text { Répartition des titres } \\
\text { Coefficient de Gini des titres } \\
\text { Distribution des pourcentages de victoires }\end{array}$ & MLB, NHLNBA, NFL & $1901-1990$ \\
\hline Butler (1995) & Coefficient de corrélation des pourcentages de victoires & MLB & $1947-1991$ \\
\hline & & NHL & 1930-1941 \\
\hline & Répartition des titres & NHL & $1930-1941$ \\
\hline Lavoie (1997) & Coefficient de corrélation des rangs & NHL & $1987-1995$ \\
\hline Bourg (1998) & Répartition des titres & Ligue 1 & $1946-1997$ \\
\hline $\begin{array}{l}\text { Hoehn \& Szymanski } \\
\text { (1999) }\end{array}$ & $\begin{array}{c}\text { Nombre de clubs qualifiés dans les compétitions euro- } \\
\text { péennes/nombre maximal possible } \\
\text { Part des qualifications européennes des trois et sept } \\
\text { meilleures équipes }\end{array}$ & $\begin{array}{c}\text { Serie A, Bundesliga } 1, \text { Liga } 1, \\
\text { Premier League }\end{array}$ & $1988-1997$ \\
\hline $\begin{array}{l}\text { Szymanski \& } \\
\text { Kuypers (1999) }\end{array}$ & Courbe de Lorenz des titres & $\begin{array}{l}\text { Liga 1, Serie A, Eredivisie, Premier } \\
\text { League, Scottish Premier League }\end{array}$ & $1946-1998$ \\
\hline
\end{tabular}

l'autre. Il peut s'agir simplement de comptabiliser sur une période déterminée le nombre de fois où les équipes ont eu accès au titre de champion ou aux premières places (qualificatives pour la Ligue des champions par exemple si l'on considère les championnats européens de football) et de comparer les résultats obtenus pour différentes équipes et/ou de déceler une éventuelle persistance. Une alternative repose sur le calcul de coefficients de corrélation des résultats sportifs annuels des équipes. Pour de tels coefficients, la valeur obtenue est comprise entre -1 et 1 . Un coefficient proche de 1 signifie une forte reproduction des résultats d'une année sur l'autre. À l'opposé, un coefficient proche de -1 rend compte d'une inversion quasi totale des résultats d'une saison sur l'autre. Le principal coefficient qui peut être relevé est le coefficient de corrélation classique (Tab. 5). Il est défini par :

$$
\rho(x, y)=\operatorname{cov}(x, y) /\left(\sigma_{x} \sigma_{y}\right)
$$

où cov est la covariance (égale à $\left.\sum[(x-\mathrm{E}(x))(y-\mathrm{E}(y))]\right)$ et $\sigma$ l'écart-type.

\section{La remise en cause de l'approche « Noll-Scully »}

La revue de littérature sur l'EC laisse apparaître une grande diversité de mesures pour l'incertitude en cours de saison et sur plusieurs exercices tandis que l'incertitude sur l'issue des matchs est aujourd'hui régulièrement appréhendée via les cotes des équipes dans les jeux de paris. Concernant l'EC au terme d'une saison, l'approche «Noll-Scully » ou NSCB est largement développée. La suite du propos s'attache à cette dernière. Après avoir défini précisément la NSCB, un retour sur les manques relevés par Michie et Oughton (2004) est opéré. Nous proposons ensuite de pointer d'autres problèmes, non mentionnés par Michie et Oughton inhérents à la 
construction même de l'indicateur proposé par Noll et Scully.

\subsection{La définition de « l'approche Noll-Scully »}

La NSCB correspond au rapprochement entre la mesure effective de l'écart-type des pourcentages de victoires de la saison avec sa valeur théorique (qui est représentative d'un championnat parfaitement équilibré) tout en tenant compte du nombre de rencontres. Dans un premier temps, il convient donc de déterminer les pourcentages de victoires de chacune des équipes ayant participé à la compétition pour, ensuite, estimer l'écart-type des pourcentages de victoires. Dans un second temps, on évalue quelle serait la mesure de cet écart-type si toutes les équipes étaient de forces égales (Rouger, 2000, p. 8). Cette valeur théorique est calculée de la manière suivante (Fort \& Quirk, 1995, p. 1267) :

Considérons une saison de championnat où chaque équipe joue $m$ rencontres.

Si toutes les équipes sont de forces égales alors la probabilité qu'a chaque équipe de gagner chaque match, en l'absence de possibilité de match nul, est $p=0,5$.

Soit $x$ le nombre de matchs gagnés par une équipe au cours de la saison.

$x$ est une variable aléatoire discrète car elle prend uniquement des valeurs entières donc bien séparées (par opposition à variable continue).

En outre, $x$ suit une loi binomiale $\mathrm{B}(m, p)$ car on renouvelle $m$ fois de manière indépendante une épreuve de Bernoulli de paramètre $p$ (expérience aléatoire à deux issues possibles, «succès » et «échec », la probabilité d'un succès étant $p$, celle d'un échec étant $q=1-p$ ).

Par conséquent, l'espérance de $x$ est donnée par $\mathrm{E}(x)=$ $m p=0,5 m$ et la variance de $x$ par $\mathrm{V}(x)=m p q=m p$ $(1-p)=0,25 m$.

Le pourcentage de victoires $w$ de chaque équipe s'obtient avec $w=x / m$, où $\mathrm{E}(w)=\mathrm{E}(x) / m=0,5 m /$ $m=0,5$, et $\mathrm{V}(w)=\mathrm{V}(x) / m^{2}=0,25 m / m^{2}=0,25 / m$.

L'écart-type étant la racine carrée de la variance, l'écart-type des pourcentages de victoires idéal théorique est égal à :

$$
\sigma(w)=0,5 / \sqrt{m}
$$

Dès lors, la NSCB se définit comme le rapport de l'écart-type des pourcentages de victoires effectif sur l'écart-type idéal théorique. Selon cette définition largement admise et utilisée par la communauté scientifique, la compétition sera d'autant plus vive que ce rapport sera voisin de zéro et ainsi la compétition sera identifiable à une situation idéale. À l'opposé, le championnat sera d'autant moins incertain que cette valeur sera élevée. Ainsi, cet indicateur est supposé permettre la mesure de l'évolution de la concurrence à l'intérieur d'une même compétition et de comparer les degrés d'incertitude de plusieurs championnats entre eux (Bourg, 2004).

\subsection{Les limites mises en évidence par Michie et Oughton (2004)}

Michie et Oughton (2004, p. 7) considèrent que la NSCB souffre de trois limites majeures. La première est qu'elle n'est pas la plus appropriée pour mesurer l'EC dans les ligues de football où les matchs nuls sont possibles et le nombre de victoires par saison varie. De plus, selon les deux auteurs anglais : «Un second problème est que le SDW (Standard Deviation of Win percentages) est basé sur l'écart-type des pourcentages de victoires par rapport à la moyenne, qui est une mesure de dispersion ou de variation, cependant, cette mesure ne prend pas en compte d'autres aspects de la distribution des pourcentages de victoires, tels que, si oui ou non c'est toujours le même groupe de clubs qui gagnent (c'est-à-dire la domination). » Les auteurs précisent que Humphreys (2002) a montré que l'indicateur SDW est insensible à la domination. Néanmoins, calculé sur la base des pourcentages de victoires de chaque équipe sur plusieurs saisons (ce qui suggère la stabilité des équipes d'une saison sur l'autre et donc la fermeture de la ligue), le SDW va traduire la domination éventuelle d'une ou quelques équipes. En effet :

- plus la valeur trouvée sera proche de la moyenne des valeurs annuelles, plus on retrouvera les mêmes équipes aux mêmes places (l'égalité entre valeur trouvée et moyenne des valeurs annuelles marquant la stabilité totale du classement) ;

- à l'inverse, plus la valeur trouvée sera éloignée de la moyenne des valeurs annuelles, moins on retrouvera les mêmes équipes aux mêmes places.

Dans tous les cas, la valeur trouvée sera inférieure ou égale à la moyenne des valeurs annuelles.

De plus, Michie et Oughton (p. 7) relèvent : «Un dernier problème est que la mesure est peu intuitive puisque l'écart-type des pourcentages de victoires n'est pas facile à interpréter. En particulier, les mesures de l'équilibre compétitif basées sur l'écart-type des pourcentages de victoires ne capturent pas nettement l'équilibre des forces compétitives entre les clubs meneurs (ou les prétendants pour le titre) et le reste, et il s'agit d'une des caractéristiques qui importe aux fans en termes d'incertitude du résultat sur la saison. » Pour corroborer le propos des deux anglais, on peut souligner qu'à une même valeur de la NSCB peuvent être associés :

- un championnat équilibré pour les premières et dernières places (celles à enjeux sauf pour les secondes lorsque le championnat est fermé) mais déséquilibré sur les autres ;

- un championnat déséquilibré pour les premières et dernières places mais équilibré sur les autres équipes situées dans le «ventre mou» de la compétition. 
Or, le premier cas de figure apparait clairement plus désirable que le second même si l'idéal demeure un championnat où chaque équipe a un enjeu à défendre.

Afin de remédier aux manques de la NSCB, Michie et Oughton (pp. 7-8) soumettent trois indicateurs de concentration pour mesurer l'EC au terme d'une saison :

1) le ratio de concentration de points des cinq premiers clubs du championnat (C5) et l'indice d'EC qui en découle (C5ICB) ;

2) l'indice d'Herfindahl $(\mathrm{H})$ et celui d'EC qui en résulte (HICB) ;

3) la courbe d'équilibre annuel de Lorenz.

- Le C5ICB : les cinq premiers sont-ils au-dessus du lot ?

Le ratio C5 mesure la part des points pris par les cinq premiers clubs du championnat par rapport aux points attribués au total. Il présente l'inconvénient de dépendre du nombre d'équipes en compétition, ce qui fausse les comparaisons temporelles dans le cas où la ligue a subi un changement d'effectif au cours de la période d'étude. Pour remédier à cela, Michie et Oughton (2004, p. 11) proposent l'indice C5ICB qui correspond à :

$$
[\mathrm{C} 5 /(5 / N)] * 100
$$

avec $N$ le nombre d'équipes en compétition.

Ainsi, il est possible de comparer deux éditions du championnat pour lesquelles le nombre d'équipes a été différent.

- Le HICB : toutes les équipes se tiennent-elles?

Le ratio C5 ignore les inégalités à l'intérieur des cinq premières places du classement, de même qu'il ne rend pas compte des inégalités entre les équipes classées de la sixième à la dernière place. L'indice d'Herfindahl comble ce manque. En effet, il s'agit de la somme des carrés des parts de points $\left(\mathrm{H}=\sum s_{i}^{2}\right)$. Par conséquent, l'indice d'Herfindahl capture les inégalités entre tous les clubs du championnat. Néanmoins, comme le ratio C5, il dépend du nombre d'équipes en compétition, c'est pourquoi Michie et Oughton (2004, p. 14) calcule l'indice HICB qui est égal à :

$$
[\mathrm{H} /(1 / N)] * 100 .
$$

Le HICB présente une limite en commun avec la NSCB dans la mesure où, à une même valeur de l'indicateur, peuvent être associés un championnat équilibré pour les premières et dernières places mais déséquilibré sur les autres ou, au contraire, un championnat déséquilibré pour les premières et dernières places mais équilibré sur les autres.

- La courbe d'équilibre annuel de Lorenz : est-on éloigné de la situation idéale?

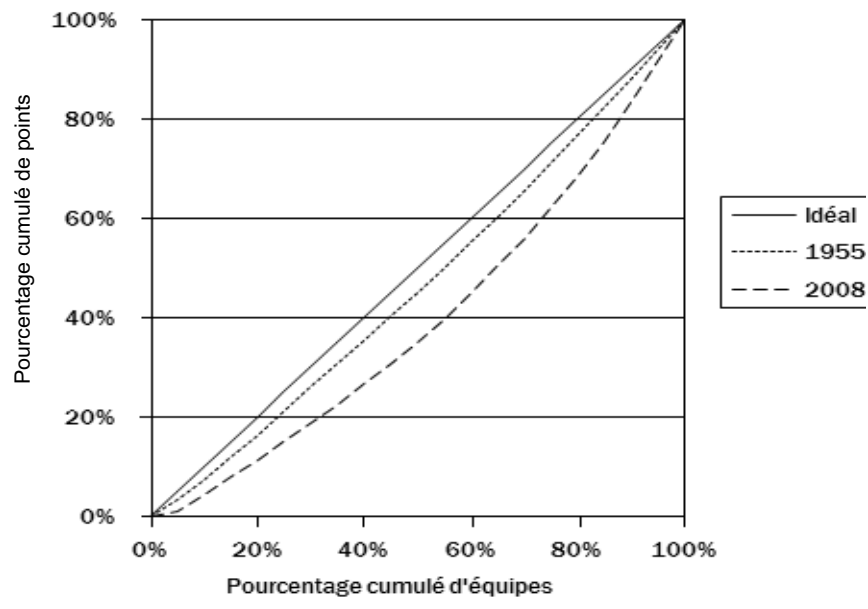

Fig. 1. Courbes de Lorenz pour les saisons 1954-1955 et 2007-2008 de Premier League anglaise de football.

La courbe d'équilibre annuel de Lorenz se construit en additionnant la part de points pris par chaque pourcentage de l'effectif en partant de l'équipe qui a acquis le moins de points pour finir par l'équipe qui en a conquis le plus. La courbe ainsi obtenue est comparée à la situation idéale où la part de points pris par chaque pourcentage de l'effectif est identique. Graphiquement, à l'axe des abscisses correspond le cumul du pourcentage de clubs ; à l'axe des ordonnées le cumul du pourcentage de points (l'échelle étant la même pour un pourcentage donné en abscisses et en ordonnées). La situation idéale est représentée par une droite passant par l'origine et le point pour lequel le cumul de toutes les équipes correspond à $100 \%$ de points pris au classement. Plus la courbe obtenue est éloignée de la droite représentative de la situation idéale, plus la compétition est déséquilibrée (voir Fig. 1 indiquant que la saison 2007-2008 a été moins équilibrée que la saison 19541955 en Premier League anglaise de football).

La courbe d'équilibre annuel de Lorenz dispose de l'avantage d'être indépendante du nombre d'équipes. Elle est utilisée pour calculer le coefficient de Gini. Celui-ci représente le rapport entre l'aire de la zone entre la ligne d'égalité parfaite et la courbe de Lorenz, et l'aire de la zone sous la ligne d'égalité parfaite. Le coefficient de Gini souffre du même défaut que la NSCB et le HICB (une même valeur de l'indicateur peut correspondre à des situations très différentes quant aux places concernées par une proximité des équipes) de sorte que les variables proposées par Michie et Oughton ne sont pas pleinement satisfaisantes. De surcroît, les deux auteurs ne questionnent pas la logique même de la construction de la NSCB. 
Tableau 6. Écart-type des pourcentages de points des cinq principaux championnats européens de football à différents moments de la saison sur la période 2004-2009.

\begin{tabular}{|l|c|c|c|c|}
\hline & Tiers & Moitié & Deux tiers & Fin \\
\hline Bundesliga 1 & $15,5 \%$ & $15,2 \%$ & $13,9 \%$ & $13,0 \%$ \\
\hline Liga 1 & $16,0 \%$ & $14,9 \%$ & $13,0 \%$ & $12,2 \%$ \\
\hline Ligue 1 & $14,8 \%$ & $12,8 \%$ & $11,3 \%$ & $10,6 \%$ \\
\hline Premier League & $17,5 \%$ & $16,5 \%$ & $15,8 \%$ & $15,3 \%$ \\
\hline Serie A & $17,1 \%$ & $16,2 \%$ & $15,3 \%$ & $13,8 \%$ \\
\hline Moyenne & $16,2 \%$ & $15,1 \%$ & $13,8 \%$ & $13,0 \%$ \\
\hline
\end{tabular}

\subsection{Les problèmes posés par la construction de « l'approche Noll-Scully »}

La NSCB a été proposée par Noll avec une visée précise : intégrer l'influence du nombre de matchs sur le pourcentage de victoires des équipes afin de pouvoir comparer des championnats ne comptant pas le même nombre de rencontres. Il en découle une formulation s'appuyant sur la confrontation entre l'écart-type réel et l'écart-type théorique représentatif d'un championnat parfaitement équilibré compte tenu du nombre de matchs joués par équipe. Or ce principe fondateur pose problème.

\subsubsection{Les problèmes liés à l'objectif}

Noll a préconisé la NSCB avec pour objectif d'incorporer l'influence du nombre de matchs. Or, cette prise en considération constitue précisément une faiblesse de l'indicateur. Pour la comprendre, il est judicieux de l'illustrer à travers l'exemple d'une ligue à quatre équipes avec matchs aller-retour dont la distribution des pourcentages de victoires est la même à mi-championnat (trois matchs) et en fin de saison (six matchs) : $100 \%$ pour la première équipe, $67 \%$ pour la seconde, $33 \%$ pour la troisième et $0 \%$ pour la quatrième : chaque équipe bat tous ses poursuivants. À mi-championnat, la NSCB est égale à 1,29 alors qu'en fin de saison, elle est égale à 1,83. Pourtant, il est évident que le championnat n'était pas plus équilibré à mi-parcours qu'à sa terminaison, l'écart-type du pourcentage de victoires étant à chaque fois égal à 37,3\%. Par conséquent, si l'intention de départ de Noll était louable, elle mène à la faillite de l'indicateur qui ne permet pas de comparer l'EC d'un championnat à différents moments de la saison, pas plus que ceux de championnats dont le nombre de matchs diffère.

Comparons la situation précédente en fin de championnat à celle d'un championnat à huit équipes avec, à nouveau, matchs aller-retour et dont la distribution des pourcentages de victoires est la suivante : $100 \%, 86 \%$, $71 \%, 57 \%, 43 \%, 29 \%, 14 \%$ et $0 \%$. L'écart-type du pourcentage de victoires pour le second championnat est égal à $32,7 \%$, inférieur à celui du premier $(37,3 \%)$. À l'opposé, sa NSCB est supérieure (égale à 2,45 au lieu de
1,83). Pourtant, on est exactement dans la même configuration : chaque équipe bat tous ses poursuivants! Dès lors, là encore, la NSCB n'est pas satisfaisante, pas plus que l'écart-type des pourcentages de victoires. Ce dernier diminue avec le nombre d'équipes mais pas obligatoirement avec le nombre de matchs a priori, car la valeur de $32,7 \%$ obtenue pour un championnat totalement déséquilibré à huit équipes est valable aussi bien avec 7 matchs qu'avec 14 matchs. En réalité, pour l'écart-type des pourcentages de points, l'observation des cinq principaux championnats européens de football sur la période 2004-2009 (Tab. 6) montre qu'il diminue au fil de la saison donc bel et bien avec le nombre de matchs, le nombre d'équipes étant donné. L'écart-type baisse donc à la fois avec le nombre d'équipes ET le nombre de matchs.

$\mathrm{Au}$ final, on a la confirmation que la comparaison des écarts-type des pourcentages de points et a fortiori de ceux des pourcentages de victoires de championnats dont le nombre de matchs diffère n'est pas pertinente. Par conséquent, Noll avait pris appui sur une faiblesse valide de l'écart-type des pourcentages de victoires pour construire la NSCB. Néanmoins, des lacunes subsistent qui tiennent en fait à sa formulation même.

\subsubsection{Les problèmes liés à la formulation}

La NSCB confronte l'écart-type des pourcentages de victoires effectif à l'écart-type théorique. Ce dernier constitue une valeur idéale, correspondant à une compétition parfaitement équilibrée. Compte tenu de la formulation de l'écart-type théorique $(\sigma(w)=0,5 / \sqrt{m})$, plus il y a de matchs, moins il est élevé. Or, dans la réalité, la compétition n'est pas parfaitement équilibrée. En définitive, plus il y a de matchs, plus la NSCB risque d'être élevée (puisque l'écart-type théorique est au dénominateur de l'indicateur) sans pour autant qu'un déséquilibre ne se soit créé en cours de saison. Aussi, on peut lui préférer l'écart-type des pourcentages de victoires ou de points. Par rapport à la NSCB, il minimise la différence de valeurs, à configuration de distribution de pourcentages de victoires ou de points équivalente, entre deux compétitions ne comptant pas les mêmes nombres d'équipes et de matchs. En effet, si l'on reprend l'exemple 
de championnats où chaque équipe bat tous ses poursuivants, les rapports entre les écarts-type des pourcentages de points de deux compétitions à configurations différentes sont plus faibles que les rapports entre leurs NSCB :

- 1,14 pour les écarts-type $(0,373 / 0,327)$ et 1,34 pour les NSCB $(2,45 / 1,83)$ pour le passage de 4 à 8 équipes et de 6 à 14 matchs ;

- 1,07 pour les écarts-type $(0,327 / 0,305)$ et 1,45 pour les NSCB $(3,56 / 2,45)$ pour le passage de 8 à 18 équipes et de 14 à 34 matchs ;

- 1,01 pour les écarts-type $(0,305 / 0,303)$ et 1,05 pour les NSCB $(3,74 / 3,56)$ pour le passage de 18 à 20 équipes et de 34 à 38 matchs.

Toutefois, l'écart-type des pourcentages de victoires ou de points ne gomme pas la différence de valeurs et n'est donc - lui non plus - pas pleinement satisfaisant.

\section{Discussion : vers un indicateur idéal ?}

Si la NSCB est la mesure la plus utilisée dans la littérature pour évaluer l'EC au terme d'une saison, elle apparaît en réalité moins pertinente que le simple calcul de l'écart-type. Ce dernier est le plus proche de la réalité de l'équilibre entre équipes sans pour autant éviter certaines faiblesses. En effet, il a été mis en évidence que l'écart-type diminue mécaniquement avec le nombre de journées, ce qui devrait être intégré pour pouvoir comparer des compétitions ne comptant pas le même nombre de journées. En outre, à une même valeur de l'indicateur peuvent correspondre deux configurations de championnats différentes : une avec beaucoup d'incertitude car les équipes proches le sont sur des places à enjeux sportifs, une autre avec peu d'incertitude car les équipes proches le sont sur des places sans enjeux sportifs. De fait, un aspect prépondérant à incorporer réside dans les enjeux sportifs (Andreff, 2008, 2009, 2010, 2011).

Par conséquent, LA «bonne » mesure de l'EC au terme d'une saison devrait prendre en considération les enjeux tout en tenant compte de manière appropriée du nombre de journées alors que dans la NSCB, sa présence n'est pas adaptée. En d'autres termes, il conviendrait de pondérer la mesure de l'EC au terme d'une saison de manière à valoriser la proximité des équipes sur des places à enjeux et à intégrer de manière précise l'impact du nombre de journées sur la valeur de l'indicateur. La détermination de la formule d'une telle mesure est extrêmement compliquée. De surcroît, une question émerge avant même d'envisager une telle détermination : mesurer l'EC au terme d'une saison est-il pertinent ?

Considérons la situation où les deux premières équipes d'un championnat sont séparées d'un point au classement final. À première vue, l'EC et donc l'incertitude sont très forts sur des places avec d'importants enjeux sportifs. Or, cette photo finale est susceptible de cacher deux scénarios très différents : l'un où le premier du championnat était assuré de finir en tête bien avant la fin de la saison et s'est autorisé de « laisser filer » les derniers matchs, permettant à son dauphin de revenir à proximité de lui au classement ; un second scénario où les deux premiers ont été au coude à coude lors des dernières journées de championnat et où l'identité du premier n'a été connue qu'à la toute fin de la dernière journée. Mesurer l'EC au terme d'une saison - mesure statique - ne rend pas compte de la dynamique d'évolution du classement au cours des dernières journées qui semble pourtant pouvoir constituer un facteur de demande du public.

Les mesures en cours de saison peuvent remédier à cet inconvénient. Dans la littérature, elles ont reçu une attention moins poussée que celles au terme d'une saison. Pourtant, un facteur explicatif de la demande du public peut tenir au renouvellement du classement en cours de saison ou, à tout le moins, au maintien d'écarts serrés entre les équipes jusqu'à la fin du championnat. Ceci est particulièrement vrai pour les places à enjeux aussi bien en haut qu'en bas du classement. Par conséquent, la détermination du degré d'incertitude du résultat des championnats peut utilement être éclairée par une approche dynamique journée après journée. En outre, l'attrait pour une compétition plutôt qu'une autre peut être lié aux caractéristiques des matchs proposés, en particulier au maintien plus long de l'incertitude du résultat ou à la plus forte capacité à proposer des rebondissements pendant les rencontres. Dès lors, des mesures intra-championnat et intra-match constituent des pistes intéressantes pour mieux appréhender l'attractivité des compétitions.

Enfin, une ultime interrogation plus fondamentale doit être soulevée : faut-il parler «seulement» d'équilibre compétitif ? Au regard des éléments développés précédemment, il semble que l'existence d'enjeux sportifs bien répartis soit au moins aussi importante qu'un équilibre entre les équipes. Il est même envisageable qu'une bonne distribution des enjeux sportifs aux différentes places du classement du championnat puisse partiellement contrebalancer un équilibre perfectible entre les équipes et générer une incertitude du résultat pour une majorité d'équipes indépendamment d'un équilibre entre chacune d'entre elles. Dans cette optique, Kringstad et Gerrard (2004) proposent la notion d'intensité compétitive (competitive intensity), indiquant qu'au-delà d'un équilibre des forces de jeu, le public va être intéressé par les prix distribués. Partant, l'intensité compétitive se rapporte bien aux enjeux sportifs qui, outre la victoire, peuvent prendre la forme d'une sélection européenne, d'une relégation en division inférieure... (Cavagnac \& Gouguet, 2006). De surcroît, l'intensité compétitive paraît devoir intégrer une autre variable omise dans l'EC : les rebondissements en cours de compétition (Scelles, 2009, 2010 ; Scelles, Desbordes \& Durand, 2011 ; Scelles \& Durand, 2007, 2010). Dès 1964, Neale (p. 3) avait mis en exergue l'importance des 
rebondissements à travers le League Standing Effect : «Il y a excitation dans les changements quotidiens des classements ou dans les changements quotidiens dans les possibilités de changements des classements. Plus les classements sont serrés, et à l'intérieur de n'importe quelle fourchette de classements plus les classements changent fréquemment, plus les recettes aux guichets seront larges. » Cette piste d'intensité compétitive à trois entrées (enjeux sportifs, incertitude du résultat, rebondissements) parait prometteuse pour pallier aux faiblesses de l'EC.

\section{Conclusion}

Si l'équilibre compétitif a fait l'objet de nombreux travaux - à la base d'une multitude de mesures différentes de l'incertitude du résultat - un certain nombre de lacunes ont été identifiées dans cette contribution. Un point important réside dans le manque de remise en cause de 《l'approche Noll-Scully », régulièrement utilisée sans que sa construction ne soit interrogée. Si Michie et Oughton (2004) critiquent la pertinence de la mesure, ils ne questionnent pas en profondeur son élaboration et proposent des indicateurs de remplacement eux-mêmes sujets à discussion. Notre analyse permet d'exposer les problèmes de construction de 《l'approche Noll-Scully » tant au niveau de son objectif (incorporer l'influence du nombre de matchs, incorporation effectivement souhaitable mais réalisée de manière inappropriée) que de sa définition (qui prend appui sur une valeur théorique représentative d'un championnat parfaitement équilibré, hypothèse totalement irréaliste). Au-delà, l'article relève une faiblesse majeure de l'EC, également pointée par Andreff dans ses développements récents (2008, 2009, 2010, 2011) : l'absence de prise en considération des enjeux sportifs. La notion d'intensité compétitive proposée par Kringstad et Gerrard (2004) permet de pallier à ce manque en pointant la nécessité d'intégrer les prix distribués pour évaluer le niveau d'incertitude d'une épreuve sportive. Elle semble pouvoir être prolongée à travers l'incorporation des rebondissements en cours de compétition dont Neale mentionnait l'importance dès 1964 via ce qu'il a qualifié de League Standing Effect. En tout état de cause, l'intensité compétitive parait pouvoir se substituer utilement à l'EC dans les travaux d'économie du sport professionnel sur les questions d'incertitude du résultat et de lien avec la demande du public. Il reste à tester son impact réel sur cette demande.

\section{Références}

Andreff, W. (2008). Équilibre compétitif et contrainte budgétaire dans une ligue de sport professionnel : Vers une meilleure gouvernance du football français. La lettre de l'AFSE, 72, 1-26.
Andreff, W. (2009). Équilibre compétitif et contrainte budgétaire dans une ligue de sport professionnel. Revue économique, 60(3), 591-633.

Andreff, W. (2010). Économie internationale du sport. Grenoble : Presses Universitaires de Grenoble.

Andreff, W. (2011). Événementiel sportif, impact économique et régulation. In M. Desbordes \& A. Richelieu (Eds.), Néomarketing du sport: Regards croisés entre Europe et Amérique du Nord (pp. 67-90). Bruxelles : De Boeck.

Baimbridge, M., Cameron, S., \& Dawson, P. (1996). Satellite television and the demand for football: A whole new ball game? Scottish Journal of Political Economy, 43(3), 317333.

Balfour, A., \& Porter, P. (1991). The reserve clause in professional sports: Legality and effect on competitive balance. Labor Law Journal, 42(1), 8-18.

Baranzini, A., Ramirez, J., \& Weber, S. (2008). The demand for football in Switzerland: An empirical estimation (Cahier de recherche). Retrieved December 22, 2008, from Genève, Haute école spécialisée de Suisse occidentale Web Site: http://www.hes-so.ch/documents/showFile.asp?ID=2693.

Barget, É., \& Rouger, A. (2000). De l'utilité de la mesure de l'équilibre compétitif. In J.J. Gouguet \& D. Primault (Eds.), Reflets et perspectives de la vie économique, 39 (23) (pp. 61-73). Bruxelles : DeBoeck Université.

Berri, D.J., Schmidt, M.B., \& Brook, S.L. (2004). Stars at the gate: The impact of star power on NBA gate revenues. Journal of Sports Economics, 5(1), 33-50.

Berri, D.J., Schmidt, M.B., \& Brook, S.L. (2006). The wages of wins: Taking measure of the many myths in modern sport. Stanford, CA: Stanford University Press.

Borland, J. (1987). The demand for Australian rules football, Economic Record, 63(182), 220-230.

Borland, J., \& Lye, J. (1992). Attendance at Australian rules football: A panel study. Applied Economics, 24, 1053-1058.

Bourg, J.-F. (1989). Le marché du travail sportif. In W. Andreff (Ed.). Économie politique du sport (pp. 145-169). Paris : Dalloz.

Bourg, J.-F. (1998). Dualisme et rapport salarial dans le sport professionnel. In J.-F. Bourg \& J.-J. Gouguet. Analyse économique du sport (pp. 119-178). Paris : PUF.

Bourg, J.-F. (2004). Les sports collectifs professionnels en Europe : Quel modèle économique ? In J.-J. Gouguet (Ed.), Le sport professionnel après l'arrêt Bosman : Une analyse économique internationale (pp. 43-60). Limoges, France : Presses Universitaires de Limoges.

Brandes, L., \& Franck, E. (2007). Who made who? An empirical analysis of competitive balance in European soccer leagues. Eastern Economic Journal, 33(3), 379-403.

Butler, M. (1995). Competitive balance in major league baseball. American Economist, 39(2), 46-52.

Buzzacchi, L., Szymanski, S., \& Valletti, T. (2003). Equality of opportunity and equality of outcome: open leagues, closed leagues and competitive balance. Journal of Industry, Competition and Trade, 3(3), 167-186. 
Cain, L.P., \& Haddock, D.D. (2006). Research notes: Measuring parity: Tying into the idealized standard deviation. Journal of Sports Economics, 7(3), 330-338.

Cairns, J. (1987). Evaluating changes in league structure: The re-organisation of the Scottish football league. Applied Economics, 19, 259-275.

Cavagnac, M., \& Gouguet, J.-J. (2006). Talent sportif et équilibre compétitif : Une approche par la théorie des jeux. Revue Juridique et Économique du Sport, 79, 7-31.

Czarnitzki, D., \& Stadtmann, G. (2002). Uncertainty of outcome versus reputation: Empirical evidence for the first German football division. Empirical Economics, 27, 101112.

Daly, G., \& Moore, W.J. (1981). Externalities, property rights and the allocation of resources in major league baseball. Economic Inquiry, 19(1), 77-95.

Demmert, H. (1973). The economics of professional team sports. Lexington: Lexington Books.

Dosseville, F. (2007). Influence of ball type on home advantage in French professional soccer. Perceptual and Motor Skills, 104, 347-351.

Durand, C., Ravenel, L., \& Helleu, B. (2005). Basket professionnel en France: Approche stratégique et géomarketing. Voiron, France : Presses Universitaires du Sport.

Eckard, E.W. (2001). Baseball blue ribbon economic report: Solutions in search of a problem. Journal of Sports Economics, 2(3), 213-227.

Forrest, D., \& Simmons, R. (2002). Outcome uncertainty and attendance in sport: The case of English soccer. The Statistician, 51, 229-241.

Forrest, D., \& Simmons, R. (2006). New issues in attendance demand: The case of the English football league. Journal of Sports Economics, 7(3), 247-266.

Forrest, D., Simmons, R., \& Buraimo, B. (2005). Outcome uncertainty and the couch potato audience. Scottish Journal of Political Economy, 52(4), 641-661.

Fort, R., \& Maxcy, J. (2003). Competitive balance in sports leagues: An introduction. Journal of Sports Economics, 4(2), 154-160.

Fort, R., \& Quirk, J. (1995). Cross-subsidization, incentives, and outcomes in professional team sports leagues. Journal of Economic Literature, 33, 1265-1299.

García, J., \& Rodríguez, P. (2002). The determinants of football match attendance revisited: Empirical evidence from the Spanish football league, Journal of Sports Economics, 3(1), 18-38.

Gouguet, J.-J. (2004). Les deux modèles d'organisation des sports professionnels : Éléments de comparaison. In J.-J. Gouguet (Ed.), Le sport professionnel après l'arrêt Bosman : Une analyse économique internationale (pp. 1128). Limoges, France : Presses Universitaires de Limoges.

Haan, M., Koning, R.H., \& van Witteloostuijn, A. (2002). Market forces in European soccer. Research Report 02F18, University of Groningen, Groningen, Netherlands. Retrieved September 20, 2006, from http://som.eldoc.ub.rug.nl/ FILES/reports/themeF/2002/02F18/02F18.pdf.
Hart, R.A., Hutton, J., \& Sharot, T. (1975). A statistical analysis of association football attendances. Applied Statistics, 24(1), 17-27.

Helleu, B. (2007). La régulation des ligues sportives professionnelles : Aspects géographiques. Le cas du football européen (1975-2005). Thèse de doctorat STAPS, non publiée, Université de Rouen, Mont-Saint-Aignan, France.

Hill, I.R., Madura, I., \& Zuber, R.A. (1982). The short run demand for major league baseball. Atlantic Economic Journal, 20(2), 31-35.

Hoehn, T., \& Szymanski, S. (1999). The americanization of European football. Economic Policy, 28, 202-240.

Humphreys, B.R. (2002). Alternatives measures of competitive balance in sports leagues. Journal of Sports Economics, 3(2), 133-148.

Jennett, N. (1984). Attendances, uncertainty of outcome and policy in the Scottish football league. Scottish Journal of Political Economy, 31(2), 176-198.

Jones, J.C.H. (1969). The economics of the national hockey league. Canadian Journal of Economics, 2(1), 1-20.

Jones, J.C.H., \& Ferguson, D.G. (1988). Location and survival in the national hockey league. Journal of Industrial Economics, 36(4), 443-457.

Knowles, G., Sherony, K., \& Haupert, M. (1992). The demand for major league baseball: A test of the uncertainty of outcome hypothesis. The American Economist, 36(2), $72-80$.

Koning, R.H. (2000). Balance in competition in Dutch soccer. The Statistician, 49(3), 419-431.

Kringstad, M., \& Gerrard, B. (2004). The concepts of competitive balance and uncertainty of outcome. International Association of Sports Economists Conference Paper, 0412.

Kringstad, M., \& Gerrard, B. (2007). Competitive balance in a modern league structure. Résumé de communication à la conférence de la North American Society for Sport Management. Ft. Lauderdale, Florida, 30 mai-2 juin. Retrieved June 12, 2007, from http://www.nassm.com/ files/conf_abstracts/2007_1715.pdf.

Kuypers, T. (1996). The beautiful game? An econometric study of why people watch English football (Discussion Paper in Economics No. 96-01). London: University College London.

La Croix, S., \& Kawaura, A. (1999). Rule changes and competitive balance in Japanese professional baseball. Economic Inquiry, 37(2), 353-68.

Lavoie, M. (1997). Avantage numérique : L'argent et la ligue nationale de hockey. Hull, Canada: Vents d'Ouest.

Michie, J., \& Oughton, C. (2004). Competitive balance in football: Trends and effects (Research Paper 2004 No. 2). Birkbeck: University of London. Retrieved October 19, 2005, from http://www.football-research.bbk.ac.uk/docs/ competitivebalance.pdf

Neale, W.C. (1964). The peculiar economics of professional sports: A contribution to the theory of the firm in sporting competition and in market competition. The Quarterly Journal of Economics, 78(1), 1-14. 
Noll, R.G. (1974). Attendance and price setting. In R.G. Noll (Ed.), Government and the sports business (pp. 115-157). Washington, DC: The Brookings Institution.

Noll, R.G. (1988). The economics of sports leagues. In G.A. Uberstine (Ed.), Law of professional and amateur sports. Deerfield, IL: Clark Boardman.

Owen, P.D., \& Weatherston, C.R. (2004). Uncertainty of outcome and super 12 rugby union attendance: Application of a general-to-specific modeling strategy. Journal of Sports Economics, 5(4), 347-370.

Peel, D., \& Thomas, D. (1988). Outcome uncertainty and the demand for football: An analysis of match attendances in the English football league. Scottish Journal of Political Economy, 35(3), 242-249.

Peel, D., \& Thomas, D. (1992). The demand for football: Some evidence on outcome uncertainty. Empirical Economics, $17(2), 323-331$.

Peel, D., \& Thomas, D. (1997). Handicaps, outcome uncertainty and attendance demand. Applied Economics Letters, 4, 567-570.

Pinnuck, M., \& Potter, B. (2006).,Impact of on-field football success on the off-field financial performance of AFL football clubs. Accounting and Finance, 46, 499-517.

Quirk, J., \& Fort, R. (1992). Pay dirt: The business of professional team sports. Princeton, NJ: Princeton University Press.

Rascher, D. (1999). A test of the optimal positive production network externality in major league baseball. In J. Fizel, E. Gustafson \& L. Hadley (Eds.), Sports Economics: Current Research (pp. 27-45). Westport, CT: Praeger.

Rottenberg, S. (1956). The baseball players' labor market. Journal of Political Economy, 64(3), 242-258.

Rouger, A. (2000). La régulation des championnats de sports collectifs professionnels : Entre équilibre compétitif et équilibre concurrentiel. Thèse de doctorat ès sciences économiques, non publiée, Université de Limoges, Limoges, France.

Scelles, N. (2009). L'incertitude du résultat, facteur clé de succès du spectacle sportif professionnel. L'intensité compétitive des ligues: Entre impacts mesurés et effets perçus. Thèse de doctorat STAPS, Université de Caen Basse-Normandie, Caen, France.

Scelles, N. (2010). La glorieuse incertitude du sport. L'intensité compétitive des ligues professionnelles: Entre impacts mesurés et effets perçus. Sarrebruck, Allemagne : Éditions Universitaires Européennes.

Scelles, N., Desbordes, M., \& Durand, C. (2011). Marketing in sport leagues: Optimising the product design. Intra- championship competitive intensity in French football Ligue 1 and basketball Pro A. International Journal of Sport Management and Marketing, 9(1/2), 13-28.

Scelles, N., \& Durand, C. (2007). L'impact du bonus sur l'intensité compétitive intra-match: Incidence réelle et potentielle dans les championnats français de rugby, de football et de basket. Revue Européenne de Management du Sport, 18 .

Scelles, N., \& Durand, C. (2010). Incertitude du résultat et demande du public: L'intensité compétitive intra-match comme variable clé. Le cas de la Ligue des champions de l'UEFA (1955/2008). Science $\&$ Motricité, 71, 65-70.

Schmidt, M.B., \& Berri, D.J. (2001). Competitive balance and attendance: The case of major league baseball. Journal of Sports Economics, 2(2), 145-167.

Schmidt, M.B., \& Berri, D.J. (2003). On the evolution of competitive balance: The impact of an increasing global search. Economic Inquiry, 41(4), 692-704.

Scully, G.W. (1989). The business of major league baseball. Chicago: University of Chicago Press.

Siegfried, J.J., \& Hinshaw, C.E. (1979). The effect of lifting television blackouts on professional football no-shows. Journal of Economics and Business, 32(1), 1-13.

Soebbing, B.P. (2008). Competitive balance and attendance in major league baseball: An empirical test of the uncertainty of outcome hypothesis. International Journal of Sport Finance, 3, 119-126.

Szymanski, S. (2001). Income inequality, competitive balance and the attractiveness of team sports: Some evidence and a natural experiment from English soccer. The Economic Journal, 111, F69-F84.

Szymanski, S. (2003). The economic design of sporting contests. Journal of Economic Literature, 41, 1137-1187.

Szymanski, S., \& Kuypers, T. (1999). Winners and losers: The business strategy of football. London: Viking Books.

Szymanski, S., \& Smith, R. (2002). Equality of opportunity and equality of outcome: Static and dynamic competitive balance in European and North American sports leagues. In C. Barros, M. Ibrahim \& S. Szymanski (Eds.), Transatlantic Sport. Cheltenham, UK: Edward Elgar.

Utt, J., \& Fort, R. (2002). Pitfalls to measuring competitive balance with Gini coefficients. Journal of Sports Economics, 3(4), 367-373.

Vrooman, J. (1995). A general theory of professional sports leagues. Southern Economic Journal, 61(4), 971-990.

Whitney, J. (1988). Winning games versus winning championships: The economics of fan interest and team performance, Economic Inquiry, 26, 703-724. 


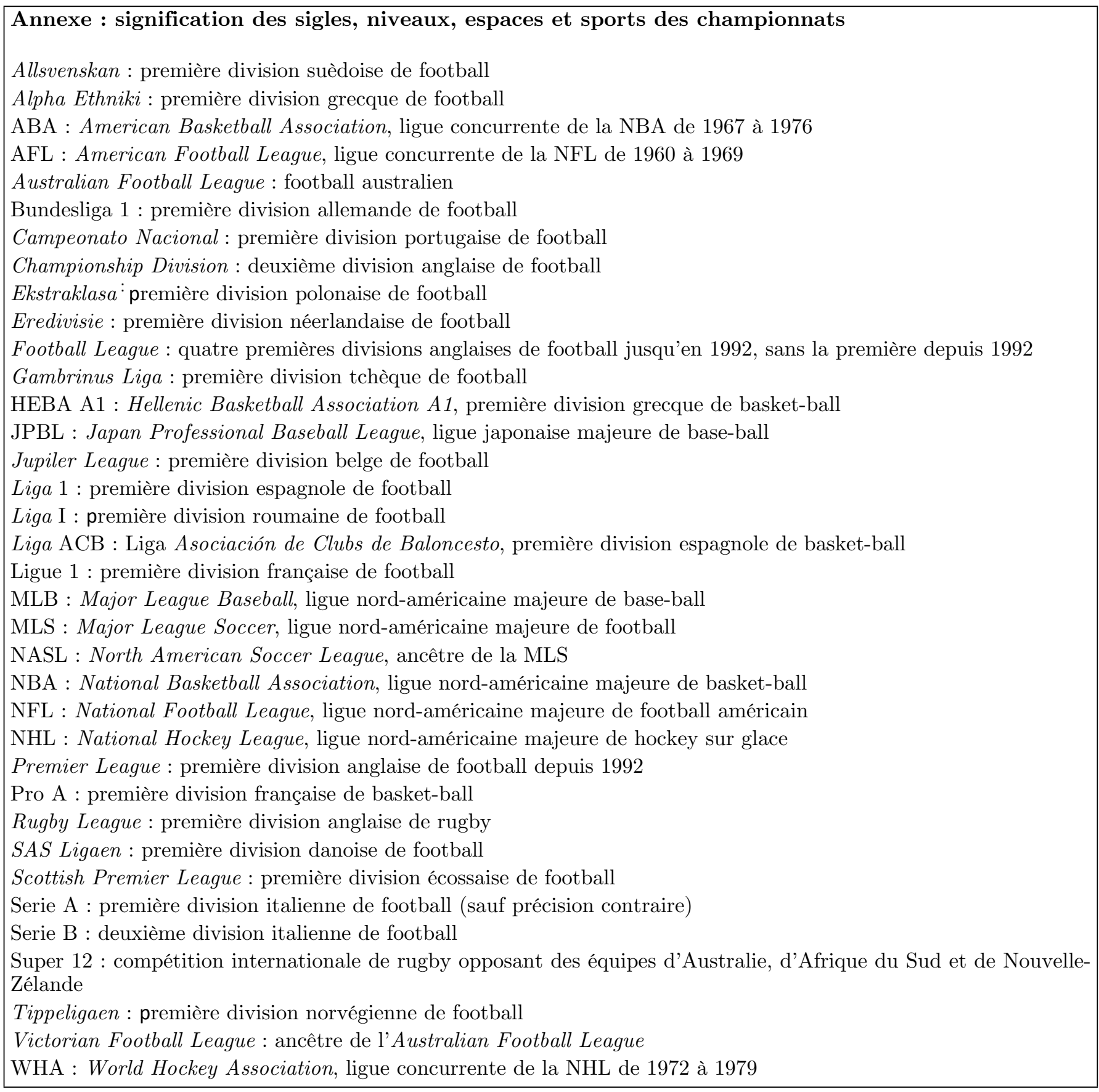

